\title{
A Cross-Sectional Study on Potential Ovarian Volume and Related Factors in Women with Polycystic Ovary Syndrome from Infertile Couples
}

\author{
Nguyen Sa Viet Le iD ${ }^{1,2}$ \\ Minh Tam Le (iD) 2,3 \\ Nguyen Dac Nguyen (iD ${ }^{2,3}$ \\ Nhu Quynh Thi Tran (D) 3 \\ Quoc Huy Vu Nguyen (D) ${ }^{2}$ \\ Thanh Ngoc Cao (iD ${ }^{2,3}$ \\ 'Department of Assisted Reproduction, \\ Hue Central Hospital, Hue, Vietnam; \\ ${ }^{2}$ Department of Obstetrics and \\ Gynecology, Hue University of Medicine \\ and Pharmacy, Hue University, Hue, \\ Vietnam; ${ }^{3}$ Center for Reproductive \\ Endocrinology and Infertility, Hue \\ University of Medicine and Pharmacy, \\ Hue University, Hue, Vietnam
}

\begin{abstract}
Purpose: This study was designed to explore the value of ovarian volume $(\mathrm{OV})$ measured by transvaginal ultrasound and its relationship with anthropometry and serum hormonal levels in a polycystic ovary syndrome (PCOS) population.

Patients and Methods: A total of 119 women with PCOS from infertile couples were recruited in this cross-sectional study. On days $2-4$ of the menstrual cycle, transvaginal ultrasound examinations were performed, and hormonal profiles were measured. PCOS diagnosis was based on the Rotterdam 2003 criteria and classified into four phenotype groups. The PCOS group (study group) and the non-PCOS group (control group) were compared.

Results: The mean age of the participants was $32.66 \pm 4.10$ years compared to $33.99 \pm 4.78$ years in 273 cases $(69.6 \%)$ without PCOS. The mean OV was statistically larger in the PCOS group than in the non-PCOS group $(7.65 \pm 3.23 \mathrm{~mL}$ vs $6.08 \pm 3.67 \mathrm{~mL}, \mathrm{p}<0.001)$ and positively correlated with serum anti-Mullerian (AMH) and luteinizing hormone (LH) levels $(\mathrm{r}=0.30 ; \mathrm{p}<0.001$ and $\mathrm{r}=0.23 ; \mathrm{p}<0.001$, respectively), and weakly and inversely correlated with age $(-0.182, \mathrm{p}<0.001)$. The area under the receiver operating characteristic (ROC) curve of OV in the diagnosis of PCOS was $0.613(0.557-0.670,95 \% \mathrm{CI})$.

Conclusion: The enlarged OV is remarkable in women with PCOS and is related to AMH and LH concentrations. Although the diagnostic potential of PCOS is substantially low, OV alone may contribute to predicting the severity of PCOS and better performance for the diagnosis of PCOS phenotypes.
\end{abstract}

Keywords: ovarian volume, anti-Mullerian hormone, PCOS phenotypes, diagnose, infertility

\section{Introduction}

Polycystic ovary syndrome (PCOS) is the most common endocrine disorder. It can affect $6 \%$ of women in the reproductive age according to the diagnostic criteria of National Institutes of Health (NIH) and up to $8-13 \%$ of these women according to the Rotterdam criteria. ${ }^{1}$ This heterogeneous disorder is characterized by hyperandrogenism, chronic anovulation, and the presence of polycystic ovaries on ultrasound. ${ }^{2}$ PCOS diagnosis is done based on the presence of oligo-anovulation and androgen excess only according to the NIH criteria, after ruling out all other reasons for anovulatory infertility. ${ }^{3}$ In 2003, the European Society for Human Reproduction and Embryology (ESHRE) and the American Society for Reproductive Medicine enlarged the consensus criteria by including polycystic ovarian morphology on ultrasoundas the third diagnostic criterion and further requires the presence of at least two of three criteria for
Correspondence: Minh Tam Le

Email leminhtam@huemed-univ.edu.vn 
a diagnosis of PCOS. ${ }^{4}$ The Rotterdam 2003 also expanded the NIH 1990 definition creating two new phenotypes. Classical phenotypes include phenotype A (full-blown syndrome PCOS); phenotype B (non-PCOS PCOS) while two additional phenotypes include phenotype $\mathrm{C}$ (ovulatory PCOS) and phenotype D (non-hyperandrogenic PCOS). More recently, the 2018 International evidence-based Guidelines on the diagnosis and management of PCOS endorsed the Rotterdam criteria for PCOS diagnosis. ${ }^{5}$ Although an ultrasound is not strictly needed in diagnosing PCOS, it is still recommended to identify the complete phenotype and may be useful for other indications in PCOS. ${ }^{5}$

Based on ultrasonographic evidence for diagnosis of PCOS, the Rotterdam consensus criteria in 2004 defined polycystic ovarian morphology (PCOM) as follicle number per ovary (FNPO) with a threshold of $\geq 12$ follicles measuring 2-9 $\mathrm{mm}$ in diameter (mean of both ovaries) and/or increased ovarian volume (OV) of $\geq 10 \mathrm{~mL}{ }^{4}$ The 2018 ESHRE PCOS guideline group suggested a threshold of $>20$ FNPO with or without an $\mathrm{OV} \geq 10 \mathrm{~mL}$ in either ovary using transvaginal ultrasound transducers with a frequency bandwidth of $8 \mathrm{MHz}$ to diagnose PCOM. ${ }^{5}$ It is worth noting that there are no changes to an $\mathrm{OV}$ of $\geq 10 \mathrm{~mL}$ the diagnosis of PCOM even imaging techniques have considerably advanced. ${ }^{6}$ However, FNPO is still recommended over OV in PCOS diagnosis over OV because of its higher predictive performance and lesser variability. ${ }^{6}$ However, the levels of intra-observer reliability and inter-reliability were higher when assessing OV than FNPO and follicle number per section. ${ }^{7}$ Therefore, $\mathrm{OV}$ is still a potential diagnostic tool for detecting PCOS. Several studies have investigated the relationship between OV and $\operatorname{PCOS},{ }^{8}$ obesity, ${ }^{9}$ insulin resistance, ${ }^{10}$ androgen activity, ${ }^{11,12}$ metabolic syndrome, ${ }^{13}$ and AMH levels ${ }^{12,14,15}$ in PCOS patients. Furthermore, to date, while an ovarian volume of $>10 \mathrm{~mL}$ is a part of the Rotterdam criteria for PCOS, several research groups have suggested various cut-off points for OV due to variation in population characteristics. The aim of this study was to examine the value of OV measured by transvaginal ultrasound and its relationship to anthropometry and serum hormonal levels in the PCOS population.

\section{Patients and Methods Study Population}

This cross-sectional study was carried out at the Hue Center for Reproductive Endocrinology and Infertility, Vietnam, from January 2019 to December 2020. Infertile women who were diagnosed with PCOS followed the Rotterdam criteria $2003^{4}$ were recruited for the study. Exclusion criteria were the presence of ovarian diseases (ovarian cyst/tumor or endometrioma), history of adnexal surgery, ovarian failure, history of hormonal contraception use, or any hormonal treatment within three months prior to enrollment. Ethical approval for this study was obtained from the Ethics Committee of the Hue University of Medicine and Pharmacy. The study was conducted in accordance with the Declaration of Helsinki. Written informed consent was obtained from all individual participants included in the study.

The sample size (n) required for this study was calculated based on the equation $\mathrm{n}=\mathrm{Z}_{\alpha / 2}{ }^{2} \times P \times(1-P) / \Delta^{2}$, where $\alpha=0.05, \Delta=0.05$, and $Z_{\alpha / 2}=1.96$. The estimated prevalence of PCOS in infertile women $(P)$ was $40.9 \%{ }^{16}$ The total sample size required in this study was 372 .

\section{Clinical and Hormonal Tests and Ultrasonography Assessment}

All participants were asked about their menstrual cycle, medical, gynecologic, and obstetric history. Oligomenorrhea was defined as having fewer than 8 menstrual cycles per year, the absence of 3-6 consecutive menstrual cycles per year or the length of menstrual cycle greater than 35 days. Clinical examinations, including measurement of body weight, height, waist and hip circumference, and evaluation for signs of hyperandrogenism were performed. Body mass index (BMI) was calculated by dividing body weight in kilograms by the square of patient height in meters. Waist and hip circumferences measurement were made on exposed skin around the abdomen at the levels of navel and pubic symphysis, respectively, in the standing position. Hirsutism was visually graded using a modification of the Ferriman and Gallwey scoring system (mFG). We defined clinical hirsutism as $\mathrm{mFG} \geq 5$ using the mFG cut-off criterion for the Asian PCOS population. ${ }^{17}$ All questions were done through a demographic questionnaire.

On day 2-4 of natural cycle or on day 2-4 of progesterone withdrawal in case of oligo or amenorrhea condition, transvaginal ultrasound examination was performed by the same experienced physician by ultrasonography (ALOKA ProSound SSD-3500, Hitachi, Japan) using a vaginal probe of $7 \mathrm{MHz}$ to evaluate each participant's antral follicle number and OV. Ovaries were scanned from the inner to the outer margin ovaries in both the transverse and sagittal planes. Three dimensions of each ovary were measured, and the total number of antral follicles that were 2-9 $\mathrm{mm}$ in diameter were counted. The OV was estimated 
for each ovary using the $\pi / 6 \times(\mathrm{D} 1 \times \mathrm{D} 2 \times \mathrm{D} 3)$ formula $\mathrm{D}$ presented the longest diameter of each ovary dimension (long, anterior-posterior, and transverse sections).

Blood samples were collected for basic hormonal testing on the same day as the ultrasound examinations were performed. Basic hormonal profiles including follicle-stimulating hormone (FSH), LH, estradiol, and prolactin were measured by an immunoradiometric assay (IRMA). AMH levels were determined by an Elecsys Roche System using electrochemiluminescence (ECLIA) technology. All tests were performed at the laboratory center of Hue University Hospital.

The PCOS diagnosis followed the Rotterdam criteria 2003 with at least two of the following three features (study group): (i) oligo-and/or anovulation, (ii) clinical and/or biochemical signs of hyperandrogenism, and/or (iii) polycystic ovaries by transvaginal ultrasound scan (more than 12 follicles in the $2-9 \mathrm{~mm}$ range in each ovary and/or $\mathrm{OV}>10 \mathrm{~mL}$ ). ${ }^{4}$ The remaining participants were infertile women without PCOS (control group). PCOS patients were classified into four phenotype groups according to the NIH consensus panel 2012. ${ }^{18}$ Phenotype A included patients with hyperandrogenism (HA), ovulatory dysfunction (OD), and polycystic ovaries (PCO) (HA $+\mathrm{OD}+\mathrm{PCO}$ ); Phenotype B included those with HA + OD; phenotype $\mathrm{C}$ included those with $\mathrm{HA}+\mathrm{PCO}$; and phenotype $\mathrm{D}$ included those with OD + PCO.

\section{Data Analysis}

The data are presented as proportions and mean \pm standard deviation (SD). Categorical data were assessed for normal distribution using the Shapiro-Wilk test. The Student's $t$-test or Mann Whitney $U$-test was used to compare the differences between the PCOS and control groups, and four phenotype groups of PCOS. Dichotomous variables were compared using two-tailed chi-square or Fisher exact tests, where appropriate. Spearman correlation was used for an analysis of the association between AMH levels and OV. Statistical significance was set at $p<0.05$. The Youden index was used to identify the best threshold values for AMH levels and OV. ROC curves were constructed to assess the diagnostic ability of AMH and OV. Sensitivity against 1 - specificity was plotted at each threshold level, and the area under the curve (AUC) was calculated. AUC represents the probability of correctly identifying controls and patients with PCOS. A value of 0.5 indicated that the result was not better than random. All statistical data were analyzed using SPSS Statistics Version 24.0 software (SPSS Inc., Chicago, IL, USA).

\section{Results}

The general characteristics, anthropometry, hormonal tests, and ultrasound scans of all recruited populations, presented in the PCOS and non-PCOS groups, are reported in Table 1. Mean age of the 119 participants was $32.66 \pm 4.10$ years compared to $33.99 \pm 4.78$ years in 273 cases without PCOS. Women with PCOS more commonly had primary infertility, higher LH and LH:FSH ratios, and lower FSH values. Serum AMH levels were 2-fold higher in the PCOS group than that in the control group $(7.35 \pm 5.17$ vs $3.48 \pm 3.17 \mathrm{ng} / \mathrm{mL})$. There were no statistically significant differences in the waist-hip ratio, mean plasma estradiol, and mean prolactin concentrations in either group. The mean OV was calculated as $7.48 \pm 3.11 \mathrm{~mL}$ in the PCOS group and $6.58 \pm 3.69 \mathrm{~mL}$ in the non-PCOS group $(\mathrm{p}<$ 0.001 ). Significant differences were observed on both sides of the ovaries.

Table 2 shows the distribution of anthropometry, clinical and hormonal profiles, and ultrasound findings among the different phenotypes. The prevalence of classical phenotype $\mathrm{A}(\mathrm{HA}+\mathrm{OD}+\mathrm{PCO})$, phenotype $\mathrm{B}(\mathrm{HA}+\mathrm{OD})$, phenotype $\mathrm{C}(\mathrm{HA}+\mathrm{PCO})$, and phenotype D (OD+PCO) were 5.04\%, $1.68 \%, 13.45 \%$, and $79.83 \%$ in women with PCOS, respectively. The four subgroups showed similar clinical, physical, and hormonal characteristics. However, $\mathrm{mFG}$ was higher in subgroup $C$ than in the other subgroups $(p<0.01)$. Serum $\mathrm{AMH}$ concentration and mean $\mathrm{OV}$ seemed to be lower in phenotype $\mathrm{B}$ than in other phenotypes, but the differences did not reach statistical significance.

The correlations between clinical characteristics and hormonal profiles with mean $\mathrm{OV}$ are shown in Table 3. AMH and $\mathrm{OV}$ were positively correlated (right $\mathrm{OV}$ : $\mathrm{r}=0.28$; left OV: $r=0.26$; mean OV: $r=0.30 ; p<0.001)$. Similarly, LH and OV had a weaker positive relationship (right OV: $\mathrm{r}=0.19$; left OV: $\mathrm{r}=0.22$; mean OV: $\mathrm{r}=0.23 ; \mathrm{p}<0.001$ ). In addition, $\mathrm{OV}$ had a weak negative relationship with age and FSH levels.

The diagnostic potency of the OV compared to the $\mathrm{AMH}$ assay was calculated using the ROC procedure. The AUC of the OV to predict PCOS was $0.613(0.557-0.670,95 \% \mathrm{CI})$ (Figure 1A). At the optimal cut-off $(4.57 \mathrm{~mL})$, the sensitivity and specificity for OV were $92.74 \%$ and $33.3 \%$, respectively. When the threshold value was set at $6.03 \mathrm{~mL}$, the sensitivity and specificity for OV were $61.3 \%$ and $50.5 \%$, respectively (Table 4). The ROC analysis of the AMH test was more informative for the diagnosis of PCOS, with an AUC of 0.787 (0.741-0.834; 95\% CI) (Figure 1B). The ideal cut-off value for AMH level was determined to be $4.195 \mathrm{ng} / \mathrm{mL}$. The 
Table I General Characteristics, Anthropometry, Hormonal Profiles and Ovarian Ultrasound Findings

\begin{tabular}{|c|c|c|c|c|}
\hline \multicolumn{2}{|l|}{ Characteristics } & $P \cos (n=119)$ & Non - PCOS $(n=273)$ & $\mathbf{p}$ \\
\hline \multicolumn{2}{|l|}{ Age (yrs) } & $32.66 \pm 4.10$ & $33.99 \pm 4.78$ & 0.042 \\
\hline \multicolumn{2}{|l|}{ Menarche (yrs) } & $13.37 \pm 0.92$ & $13.74 \pm 1.39$ & 0.002 \\
\hline \multicolumn{2}{|c|}{ Duration of infertility (yrs) } & $4.27 \pm 2.46$ & $4.09 \pm 2.80$ & 0.010 \\
\hline \multirow[t]{2}{*}{ Type of infertility } & Primary & $90(75.6)$ & $177(64.8)$ & \multirow[t]{2}{*}{0.045} \\
\hline & Secondary & $29(24.4)$ & $96(35.2)$ & \\
\hline \multicolumn{2}{|l|}{ BMI $\left(\mathrm{kg} / \mathrm{m}^{2}\right)$} & $21.31 \pm 2.80$ & $20.82 \pm 2.56$ & $<0.001$ \\
\hline \multicolumn{2}{|l|}{ mFG score } & $0.55 \pm 1.09$ & $0.14 \pm 0.46$ & $<0.001$ \\
\hline \multicolumn{2}{|l|}{ WHR } & $0.82 \pm 0.06$ & $0.83 \pm 0.07$ & 0.762 \\
\hline \multicolumn{2}{|l|}{$\mathrm{FSH}(\mathrm{mlU} / \mathrm{mL})$} & $6.07 \pm 1.46$ & $7.41 \pm 3.49$ & $<0.001$ \\
\hline \multicolumn{2}{|l|}{ LH (mlU/mL) } & $9.37 \pm 5.31$ & $6.23 \pm 3.43$ & $<0.001$ \\
\hline \multicolumn{2}{|l|}{ LH/FSH } & $1.57 \pm 0.85$ & $0.95 \pm 0.63$ & $<0.001$ \\
\hline \multicolumn{2}{|l|}{$\mathrm{E} 2(\mathrm{pg} / \mathrm{mL})$} & $43.75 \pm 26.55$ & $40.22 \pm 40.76$ & 0.137 \\
\hline \multicolumn{2}{|l|}{ Prolactin $(\mu \mathrm{IU} / \mathrm{mL})$} & $410.45 \pm 243.46$ & $432.26 \pm 318.07$ & 0.060 \\
\hline \multicolumn{2}{|l|}{ AMH (ng/mL) } & $7.35 \pm 5.17$ & $3.48 \pm 3.17$ & $<0.001$ \\
\hline \multirow[t]{3}{*}{ Ovarian volume $\left(\mathrm{cm}^{3}\right)$} & Right ovarian & $8.18 \pm 3.73$ & $6.69 \pm 4.17$ & $<0.001$ \\
\hline & Left ovarian & $6.78 \pm 3.40$ & $6.46 \pm 4.11$ & $<0.001$ \\
\hline & Mean volume & $7.48 \pm 3.11$ & $6.58 \pm 3.69$ & $<0.001$ \\
\hline
\end{tabular}

Notes: Data presented in Mean \pm standard deviation or number (percentage).

Abbreviations: PCOS, polycystic ovary syndrome; yrs, year; BMI, body mass index; mFG, modified Ferriman Gallwey; WHR, weight hip ratio; FSH, follicle stimulating hormone; $\mathrm{LH}$, luteinizing hormone; E2, estradiol; $\mathrm{AMH}$, anti-Mullerian hormone.

sensitivity and specificity at this cut-off point were $73.1 \%$ and $70.7 \%$, respectively.

\section{Discussion}

This study was designed to explore the value of OV measured by transvaginal ultrasound in the diagnosis of PCOS and its relation to anthropometry and hormonal profiles, especially AMH. This study revealed that in Vietnamese women from infertile couples with PCOS, the right $\mathrm{OV}$ was $8.25 \pm 4.03 \mathrm{~mL}$ and the left $\mathrm{OV}$ was $7.06 \pm 3.37 \mathrm{~mL}$. The mean volume of the two ovaries was $7.65 \pm 3.23 \mathrm{~mL}$; which was smaller in comparison with results from several previous studies, reported by Carmina et al $(9.6 \pm 3.2 \mathrm{~mL}),{ }^{19}$ Ndoua et al $(12.02 \pm 3.36 \mathrm{~mL}),{ }^{14}$ and Nylander et al $(9.40$ $\pm 3.62 \mathrm{~mL}){ }^{20}$ However, our findings were consistent with data of Korean PCOS patients, who had a right OV of 7.9 $\pm 3.6 \mathrm{~mL}$ and left $\mathrm{OV}$ of $6.7 \pm 3.1 \mathrm{~mL}^{12}$ In the Asian population, lower $\mathrm{OV}$ in women with PCOS was mentioned previously $^{21}$ and was supposed to differ among women in various ethnic subgroups of women. ${ }^{22,23}$ Furthermore, ovarian volume varies throughout the reproductive life of a woman, that is, it reaches its maximal size during adolescence, slowly declines during adulthood, and rapidly shrinks during menopause. ${ }^{24,25}$ Therefore, the difference in age could explain the difference in mean OV between studies.

In previous studies, a larger mean $\mathrm{OV}$ in women with PCOS was reported compared to that in the non-PCOS population. ${ }^{12,21,26,27}$ The ovaries of PCOS patients generally showed an increased number of small antral follicles and different degrees of thecal cell hyperplasia and hypertrophy, stromal hyperplasia and hypertrophy, and cortical thickening, resulting in an increase in the OV. ${ }^{28,29}$ Several studies conducted based on the Rotterdam consensus suggested a lower cut-off for OV ranging from 6.4 to $7.5 \mathrm{~mL}$ $\left(6.4 ;^{30} 6.7 ;^{27} 7.0 ;^{31,32}\right.$ and $\left.7.5 \mathrm{~mL}^{26}\right)$ to increase the sensitivity of the polycystic ovary definition. These differences could be explained by the variation in population characteristics, particularly the ethnic factors, obesity, serum insulin level, and the methods used to determine the OV. ${ }^{11,24,25,33}$ Our data revealed that the diagnostic 
Table 2 Comparison of General Characteristics, Anthropometry, Hormonal Profiles and Ovarian Ultrasound Findings Between PCOS Phenotypes

\begin{tabular}{|c|c|c|c|c|c|}
\hline Characteristics & $\begin{array}{c}\text { Phenotype A } \\
\text { (HA+OD+PCO) }\end{array}$ & $\begin{array}{c}\text { Phenotype B } \\
\text { (HA+OD) }\end{array}$ & $\begin{array}{c}\text { Phenotype C } \\
\text { (HA+PCO) }\end{array}$ & $\begin{array}{c}\text { Phenotype D } \\
\text { (OD+PCO) }\end{array}$ & $\mathbf{p}$ \\
\hline Prevalence & $6(5.04)$ & $2(1.68)$ & $16(13.45)$ & $95(79.83)$ & \\
\hline Age (yrs) & $30.50 \pm 2.43$ & $37.00 \pm 12.73$ & $32.31 \pm 4.14$ & $32.77 \pm 3.95$ & 0.433 \\
\hline Menarche (yrs) & $13.33 \pm 0.52$ & $|3.50 \pm 0.7|$ & $13.75 \pm 1.39$ & $|3.3| \pm 0.84$ & 0.042 \\
\hline Duration of infertility (yrs) & $4.00 \pm 3.03$ & $4.00 \pm 2.83$ & $2.94 \pm 2.29$ & $4.52 \pm 2.41$ & 0.017 \\
\hline BMI & $21.48 \pm 3.11$ & $20.12 \pm 0.83$ & $20.55 \pm 1.74$ & $21.45 \pm 2.95$ & 0.694 \\
\hline mFG score & $0.33 \pm 0.52$ & 0 & $2.06 \pm 1.81$ & $0.32 \pm 0.70$ & $<0.001$ \\
\hline WHR & $0.81 \pm 0.05$ & $0.77 \pm 0.07$ & $0.80 \pm 0.06$ & $0.82 \pm 0.06$ & 0.540 \\
\hline $\mathrm{FSH}(\mathrm{mlU} / \mathrm{mL})$ & $6.10 \pm 1.05$ & $6.44 \pm 2.21$ & $6.71 \pm 2.05$ & $5.95 \pm 1.35$ & 0.551 \\
\hline $\mathrm{LH}(\mathrm{mlU} / \mathrm{mL})$ & $11.75 \pm 7.72$ & $7.57 \pm 4.62$ & $10.75 \pm 5.85$ & $9.03 \pm 5.07$ & 0.539 \\
\hline E2 $(\mathrm{pg} / \mathrm{mL})$ & $57.63 \pm 41.37$ & $67.14 \pm 45.63$ & $58.39 \pm 30.29$ & $39.92 \pm 23.46$ & 0.017 \\
\hline Prolactin $(\mu \mathrm{lU} / \mathrm{mL})$ & $455.77 \pm 245.09$ & $419.25 \pm 250.67$ & $413.84 \pm 178.74$ & $406.83 \pm 255.53$ & 0.888 \\
\hline $\mathrm{AMH}(\mathrm{ng} / \mathrm{mL})$ & $7.35 \pm 6.28$ & $2.73 \pm 0.89$ & $7.94 \pm 7.33$ & $7.35 \pm 4.73$ & 0.264 \\
\hline Ovarian volume $\left(\mathrm{cm}^{3}\right)$ & $7.48 \pm 2.35$ & $3.87 \pm 1.38$ & $7.15 \pm 4.74$ & $7.61 \pm 2.82$ & 0.059 \\
\hline
\end{tabular}

Notes: Data presented in Mean \pm standard deviation or number (percentage).

Abbreviations: PCOS, polycystic ovary syndrome; yrs, year; BMI, body mass index; mFG, modified Ferriman Gallwey; WHR, weight hip ratio; FSH, follicle stimulating hormone; LH, luteinizing hormone; E2, estradiol; AMH, anti-Mullerian hormone.

Table 3 Correlation Between Anthropometry, Hormonal Profiles and Ovarian Volume

\begin{tabular}{|l|c|c|c|c|c|c|}
\hline \multirow{2}{*}{ Parameters } & \multicolumn{2}{|c|}{ Right Ovarian Volume } & \multicolumn{2}{|c|}{ Left Ovarian Volume } & \multicolumn{2}{c|}{ Mean Volume } \\
\cline { 2 - 7 } & $\mathbf{r}$ & P value & $\mathbf{r}$ & P value & r & P value \\
\hline Age $(\mathrm{yrs})$ & -0.160 & 0.001 & -0.162 & 0.001 & -0.182 & $<0.001$ \\
BMI & 0.123 & 0.015 & 0.048 & 0.343 & 0.098 & 0.054 \\
$\mathrm{mFG}$ score & 0.100 & 0.052 & 0.106 & 0.036 & 0.115 & 0.022 \\
WHR & -0.019 & 0.705 & -0.027 & 0.601 & -0.026 & 0.612 \\
FSH $(\mathrm{mUl} / \mathrm{mL})$ & -0.129 & 0.010 & -0.087 & 0.084 & -0.123 & 0.015 \\
LH $(\mathrm{mUl} / \mathrm{mL})$ & 0.193 & $<0.001$ & 0.219 & $<0.001$ & 0.233 & $<0.001$ \\
E2 $(\mathrm{pg} / \mathrm{mL})$ & -0.058 & 0.254 & 0.029 & 0.568 & -0.017 & 0.731 \\
Prolactin $(\mu \mathrm{lU} / \mathrm{mL})$ & -0.058 & 0.251 & -0.019 & 0.713 & -0.044 & 0.386 \\
AMH $(\mathrm{ng} / \mathrm{mL})$ & 0.281 & $<0.001$ & 0.263 & $<0.001$ & 0.307 & $<0.001$ \\
\hline
\end{tabular}

Abbreviations: PCOS, polycystic ovary syndrome; yrs, year; BMI, body mass index; mFG, modified Ferriman Gallwey; WHR, weight hip ratio; FSH, follicle stimulating hormone; LH, luteinizing hormone; E2, estradiol; AMH, anti-Mullerian hormone.

potential of OV was substantially low (AUC 0.613 [0.557$0.670]$ ). At the cut-off point of $6.0 \mathrm{~mL}$, a sensitivity of $61.3 \%$ and a specificity of $50.5 \%$ were obtained. The specificity of OV reached $57.5 \%$ when its cut-off value was $6.3 \mathrm{~mL}$, but the sensitivity was lower (only $57.1 \%$ ). These findings were different from several published studies that concluded that OV had satisfactory power for use in the diagnosis of PCOS..$^{21,27,31,32}$ The main reasons for this discrepancy are the differences in the study population and ethnicity. As mentioned above, our PCOS women had remarkably small ovaries and the difference range in $\mathrm{OV}$ between the PCOS and non-PCOS population was also lower (7.65 $\pm 3.23 \mathrm{~mL}$ vs $6.08 \pm 3.67 \mathrm{~mL}$, respectively).
Our study confirmed that AMH is a good diagnostic tool for PCOS. It is a much better predictor of PCOS than ovarian volume, with an AUC of 0.787 (0.741-0.834, 96\% CI). AMH threshold at $4.2 \mathrm{ng} / \mathrm{mL}$ provided the best compromise, with a sensitivity of $73.1 \%$ and specificity of $70.7 \%$. There is emerging evidence in the published literature to confirm the potential of AMH for the diagnosis of PCOS, with moderate to excellent sensitivity and specificity at cut-off points ranging from $3.94 \mathrm{ng} / \mathrm{mL}$ to $5 \mathrm{ng} / \mathrm{mL}^{20,34-37}$ A meta-analysis by Illiodromiti et al using ROC analysis figured out an AMH cutoff value of $4.7 \mathrm{ng} / \mathrm{mL}$ to obtain $82.8 \%$ sensitivity and $79.4 \%$ specificity. ${ }^{38}$ It is important to highlight that the differences in ethnicity, age of study populations, criteria used to define 

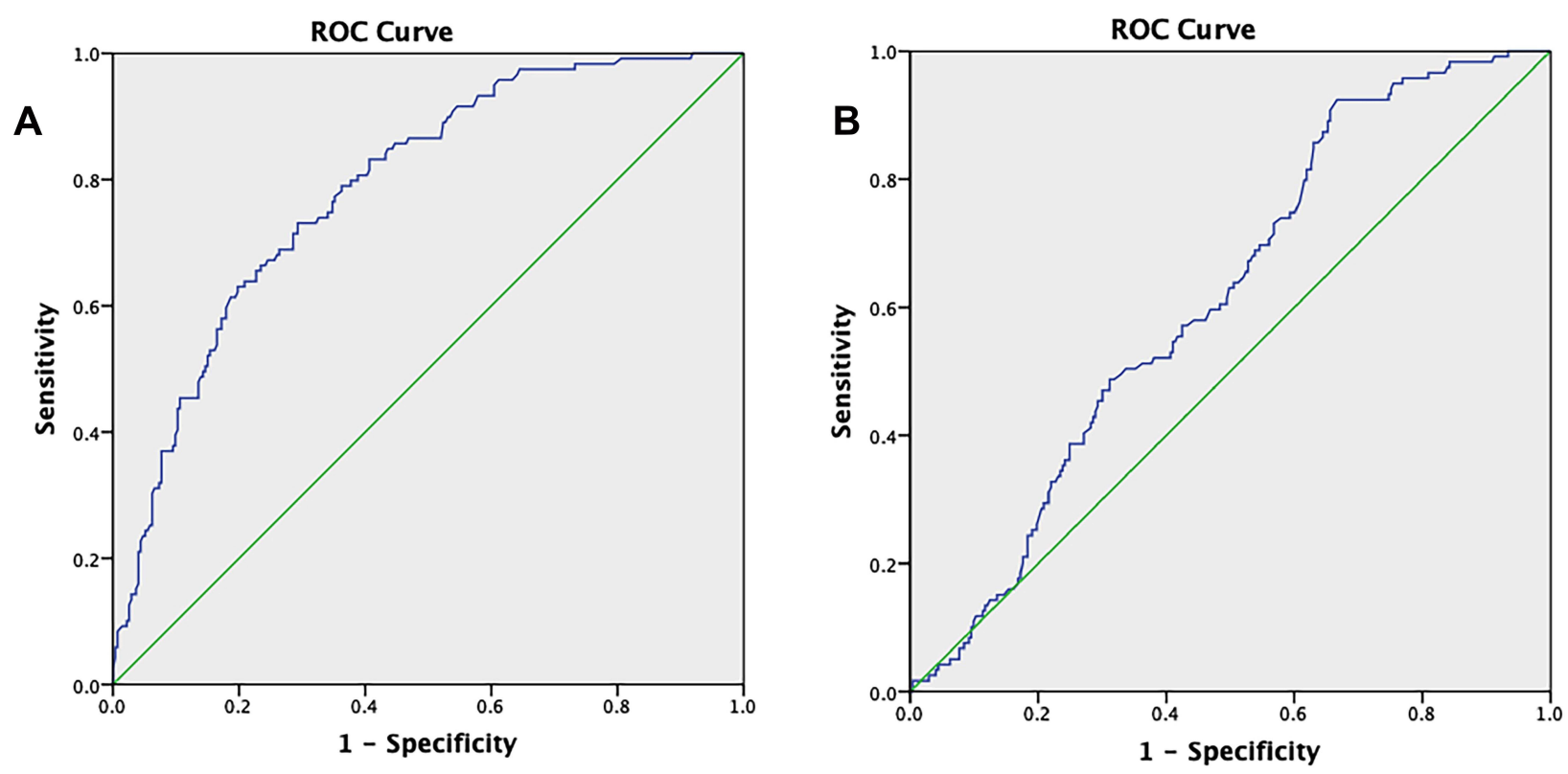

Figure I ROC curve of prediction PCOS by AMH (A) and by mean of ovarian volume (B). Diagonal segments are produced by ties.

PCOS, and type of AMH assays may influence on the results and proposed diagnostic cut-off. ${ }^{39-41}$

Published studies comparing the diagnostic ability of AMH measurement and ovarian imaging have reported conflicting results. Carmina et al carried out a retrospective matched controlled study and concluded that AMH did not appear to be as helpful as compared to FNPO and OV. ${ }^{19}$ They also assumed that FNPO was significantly sensitive in all phenotypes and was the single best criterion, supporting the essential role of ultrasound in PCOS diagnosis. ${ }^{19}$ In contrast, Wongwanaruruk et al considered AMH to be a superior predictor of ultrasonographic imaging of ovarian morphology, as the latter is dependent on the quality of ultrasound scanners and sonographer experience. ${ }^{21}$ However, the International evidence-based guideline for the assessment and management of PCOS 2018 still did not recommend the use of AMH as an alternative for the detection of PCOM or as a single diagnostic test.
The present study demonstrated a positive correlation between $\mathrm{OV}$ and serum AMH and LH levels in women with PCOS. Some other Korean authors reported this relationship to be consistent with our results. ${ }^{12,42}$ Ndoua et al demonstrated that AMH showed a good correlation with OV $(\mathrm{r}=0.625, \mathrm{p}=0.0001) .{ }^{14}$ In addition, we found a weak negative correlation between $\mathrm{OV}$ and age. This finding was also reported by Erdem et al (with $\mathrm{r}$ $\mathrm{r}-0.29, \mathrm{p}<0.05) .{ }^{43}$ As OV in women aged $25-51$ years reflects the number of primordial follicles, ${ }^{44}$ it was not difficult to explain the negative correlation between OV and age, and the positive correlation between $\mathrm{OV}$ and $\mathrm{AMH}$, as the latter was demonstrated to strongly correlate with the primordial follicle pool. ${ }^{45}$

As demonstrated in our data, there were no differences in age, BMI, WHR and hormonal profiles among four phenotypes of PCOS. Serum AMH level and mean ovarian volume were insignificantly lower in phenotype B than in other

Table 4 Diagnostic Values of Anti-Mullerian Hormone and Ovarian Volume for Polycystic Ovary Syndrome

\begin{tabular}{|l|c|c|c|c|}
\hline Parameters & Threshold & Sensitivity & Specificity & AUC, $\mathbf{p}$ \\
\hline Mean of ovarian volume & 4.575 & 0.924 & 0.333 & $0.613(0.557-0.670)$ \\
& 6.030 & 0.613 & 0.505 & $P<0.001$ \\
\hline AMH $(\mathrm{ng} / \mathrm{mL})$ & 4.195 & 0.731 & 0.707 & $\begin{array}{c}0.787(0.741-0.834) \\
P<0.001\end{array}$ \\
\hline
\end{tabular}

Abbreviations: AUC, area under curve; $\mathrm{AMH}$, anti-Mullerian hormone. 
phenotypes. In fact, previous studies showed controversial results that phenotype A was the most severe form with more frequent prevalence of obesity, hyperandrogenism, insulin resistance, deranged lipid profile, metabolic syndrome and higher LH, LH-FSH ratio compared to others; whereas Phenotype D was associated with the least severe profile. ${ }^{46,47}$ However, other studies suggested that no difference was observed in the clinical biochemical profiles of patients with different phenotypes. ${ }^{48,49}$ Similar to our ultrasound findings, Clark et al, and Sachdeva et al have also described smaller ovarian volumes in phenotype $\mathrm{B}^{46,50}$ In addition to sample size, most researchers agreed that racial differences in different study populations, again, may explain these controversies. Indeed, our results seemed to be more consistent with those from studies on Chinese populations. ${ }^{48,49}$

The strength of our study was its relatively large sample size and its comparative cross-sectional design. Notably, although measurement of OV alone was insufficient to diagnose PCOS in the Vietnamese PCOS population, its correlation with $\mathrm{AMH}$ could be a contributor to PCOS severity. However, the present study only recruited women from infertile couples, which may have resulted in selection of a relatively young age group. Therefore, neither the women diagnosed with PCOS nor the controls can be considered representative of the general population. The impact of this select study population should be considered in the drawn conclusion. Additionally, this study was performed in a single center and due to the study population included women from infertile couples, the data did not represent the general population. Thus, our conclusions must be considered in light of these limitations. Further prospective studies with agematched controls are needed for more definitive conclusions.

\section{Conclusion}

In summary, our data suggested that in women with PCOS from infertile couples, OV had a significant positive correlation with serum $\mathrm{AMH}$ and $\mathrm{LH}$ levels and a negative correlation with age. The diagnostic potential of OV was substantially low, and the measurement of OV by transvaginal ultrasound alone to diagnose PCOS should not be considered in Vietnamese infertile women. Alternatively, serum AMH levels seemed to have better performance in the diagnosis of PCOS in this population.

\section{Abbreviations}

OV, ovarian volume; PCOS, polycystic ovary syndrome; $\mathrm{AMH}$, anti-Mullerian hormone; LH, luteinizing hormone; ROC curve, receiver operating characteristic curve; NIH,
National Institutes of Health; PCOM, polycystic ovarian morphology; FNPO, follicle number per ovary; ESHRE, European Society for Human Reproduction and Embryology; BMI, body mass index; $\mathrm{mFG}$, modification of the Ferriman and Gallwey scoring system; FSH, Follicle-stimulating hormone; IRMA, immunoradiometric assay; ECLIA, electrochemiluminescence; OD, ovulatory dysfunction, $\mathrm{PCO}$, polycystic ovaries; $\mathrm{SD}$, standard deviation; AUC, area under the curve.

\section{Data Sharing Statement}

The dataset used and/or analyzed during the current study is available from the corresponding author upon reasonable request.

\section{Ethics Approval and Informed Consent}

The study was approved by the Ethics Committee of the Hue University of Medicine and Pharmacy on November 9th, 2018 (approval number H2018/432). The present study was conducted in accordance with the Declaration of Helsinki. Informed consent form was obtained for all participants prior to participation in this study.

\section{Acknowledgments}

The authors thank the staff of Hue Center for Reproductive Endocrinology and Infertility (HueCREI) for support on data recruitment.

\section{Funding}

This research did not receive any specific grant from any funding agency in the public, commercial or not-for-profit sectors.

\section{Disclosure}

The authors alone are responsible for the content and writing of this article. The authors report no conflicts of interest for this work.

\section{References}

1. Bozdag G, Mumusoglu S, Zengin D, Karabulut E, Yildiz BO. The prevalence and phenotypic features of polycystic ovary syndrome: a systematic review and meta-analysis. Hum Reprod. 2016;31 (12):2841-2855. doi:10.1093/humrep/dew218

2. Legro RS. Evaluation and treatment of polycystic ovary syndrome. [Updated 2017 Jan 11]. In: Feingold KR, Anawalt B, Boyce A, editors. Endotext. South Dartmouth (MA): MDText.com, Inc; January 11, 2017. Available from: https://www.ncbi.nlm.nih.gov/books/ NBK278959/. 
3. Zawadzki JK, Dunaif A. Diagnostic criteria for polycystic ovary syndrome: towards a rational approach. In: Dunaif A, Givens J, Haseltine F, editors. Polycystic Ovary Syndrome, 1st. Oxford, United Kingdom: Blackwell Scientific; 1992:377-384.

4. Rotterdam ESHRE/ASRM-Sponsored PCOS Consensus Workshop Group Rotterdam ESHRE/ASRM-Sponsored PCOS Consens Workshop Groupement Revised. 2003 consensus on diagnostic criteria and long-term health risks related to polycystic ovary syndrome. Revised 2003 consensus on diagnostic criteria and long-term health risks related to polycystic ovary syndrome. Fertil Steril. 2004;81(1):19-25.

5. Teede HJ, Misso ML, Costello MF, et al. Recommendations from the international evidence-based guideline for the assessment and management of polycystic ovary syndrome. Hum Reprod. 2018;33 (9):1602-1618. doi:10.1093/humrep/dey256

6. Singh AK, Singh R. Can anti-mullerian hormone replace ultrasonographic evaluation in polycystic ovary syndrome? A review of current progress. Indian J Endocrinol Metab. 2015;19(6):731-743. doi:10.4103/2230-8210.167548

7. Lujan ME, Jarrett BY, Brooks ED, et al. Updated ultrasound criteria for polycystic ovary syndrome: reliable thresholds for elevated follicle population and ovarian volume. Hum Reprod. 2013;28 (5):1361-1368. doi:10.1093/humrep/det062

8. Guandaru NM. Correlation between Ovarian Volume and Polycystic Ovarian Syndrome Using Ultrasound [Unpublished Master's dissertation]. East Africa: Aga Khan University; 2017.

9. Ashwini Sidhmalswamy G, Ghongdemath JS, Venkatesh S. Clinical, ultrasonographical and hormonal correlation in women with polycystic ovarian syndrome. Int $J$ Reprod Contracept Obstet Gynecol. 2018;7(12):5134-5139. doi:10.18203/2320-1770.ijrcog20184980

10. Reid SP, Kao CN, Pasch L, Shinkai K, Cedars MI, Huddleston HG. Ovarian morphology is associated with insulin resistance in women with polycystic ovary syndrome: a cross sectional study. Fertil Res Pract. 2017;3:8. doi:10.1186/s40738-017-0035-z

11. Turhan NO, Senoz S, Gulekli B, Ozaksit G, Oral H, Gokmen O. Clinical and endocrine features of ultrasound diagnosed polycystic ovary patients: the correlation between ovarian volume and androgen activity. $J$ Pak Med Assoc. 1993;43(1):4-6.

12. Han YS, Lee AR, Song HK, et al. Ovarian volume in Korean women with polycystic ovary syndrome and its related factors. J Menopausal Med. 2017;23(1):25-31. doi:10.6118/jmm.2017.23.1.25

13. Sipahi M, Tokgöz VY, Keskin Ö, Atasever M, Menteşe A, Demir S. Is ovarian volume a good predictor to determine metabolic syndrome development in polycystic ovary patients. J Obstet Gynaecol. 2019;39(3):372-376. doi:10.1080/01443615.2018.1522530

14. Noa Ndoua CC, Ayissi Ngah G, Mve Koh V, Belinga E, Kemfang JD, Kasia JM. Correlation between the anti-müllerian hormone and endovaginal ultrasound in the predictivity of PCOS syndrome at Chracerh. Int Reprod Contracept Obstet Gynecol. 2017;6(12):5183-5187. doi:10.18203/2320-1770.ijrcog20175224

15. Youssef H, Marei ES. Relation between anti-müllerian hormone with antral follicle count and ovarian volume in polycystic ovary syndrome. Arab J Nucl Sci Appl. 2019;52(2):84-93.

16. Baqai Z, Khanam M, Parveen S. Prevalence of PCOS in infertile patients. Med Channel. 2010;16(3):437-440.

17. Zhao X, Ni R, Li L, et al. Defining hirsutism in Chinese women: a cross-sectional study. Fertil Steril. 2011;96(3):792-796. doi:10.1016/ j.fertnstert.2011.06.040

18. National Institutes of Health. Evidence-based methodology workshop on polycystic ovary syndrome executive summary; 2012. Available from: https://prevention.nih.gov/docs/programs/pcos/FinalReport.pdf. Accessed August 18, 2021

19. Carmina E, Campagna AM, Fruzzetti F, Lobo RA. AMH measurement versus ovarian ultrasound in the diagnosis of polycystic ovary syndrome in different phenotypes. Endocr Pract. 2016;22 (3):287-293. doi:10.4158/EP15903.OR
20. Nylander M, Frøssing S, Bjerre AH, et al. Ovarian morphology in polycystic ovary syndrome: estimates from $2 \mathrm{D}$ and $3 \mathrm{D}$ ultrasound and magnetic resonance imaging and their correlation to antimüllerian hormone. Acta Radiol. 2017;58(8):997-1004. doi: $10.1177 / 0284185116676656$

21. Wongwananuruk $\mathrm{T}$, Panichyawat $\mathrm{N}$, Indhavivadhana $\mathrm{S}$, et al. Accuracy of anti-müllerian hormone and total follicles count to diagnose polycystic ovary syndrome in reproductive women. Taiwan J Obstet Gynecol. 2018;57(4):499-506. doi:10.1016/j. tjog.2018.06.004

22. Welt CK, Arason G, Gudmundsson JA, et al. Defining constant versus variable phenotypic features of women with polycystic ovary syndrome using different ethnic groups and populations. J Clin Endocrinol Metab. 2006;91(11):4361-4368. doi:10.1210/jc.2006-1191

23. Dewailly D. Diagnostic criteria for PCOS: is there a need for a rethink? Best Pract Res Clin Obstet Gynaecol. 2016;37:5-11. doi:10.1016/j.bpobgyn.2016.03.009

24. Dewailly D, Lujan ME, Carmina E, et al. Definition and significance of polycystic ovarian morphology: a task force report from the androgen excess and polycystic ovary syndrome society. Hum Reprod Update. 2014;20(3):334-352. doi:10.1093/humupd/dmt061

25. Bachanek M, Abdalla N, Cendrowski K, Sawicki W. Value of ultrasonography in the diagnosis of polycystic ovary syndrome - literature review. J Ultrasound. 2015;15(63):410-422. doi:10.15557/ JoU.2015.0038

26. Carmina E, Orio F, Palomba S, Longo RA, Lombardi G, Lobo RA. Ovarian size and blood flow in women with polycystic ovary syndrome and their correlations with endocrine parameters. Fertil Steril. 2005;84(2):413-419. doi:10.1016/j.fertnstert.2004.12.061

27. Chen Y, Li L, Chen X, et al. Ovarian volume and follicle number in the diagnosis of polycystic ovary syndrome in Chinese women. Ultrasound Obstet Gynecol. 2008;32(5):700-703. doi:10.1002/ uog.5393

28. Witchel SF, Oberfield SE, Peña AS. Polycystic ovary syndrome: pathophysiology, presentation, and treatment with emphasis on adolescent girls [presentation]. $\quad J \quad J$ Endocrinol Soc. 2019;3 (8):1545-1573. doi:10.1210/js.2019-00078

29. Rosenfield RL, Ehrmann DA. The pathogenesis of polycystic ovary syndrome (PCOS): the hypothesis of PCOS as functional ovarian hyperandrogenism revisited. Endocr Rev. 2016;37(5):467-520.

30. Köşüş N, Köşüş A, Turhan NÖ, Kamalak Z. Do threshold values of ovarian volume and follicle number for diagnosing polycystic ovarian syndrome in Turkish women differ from western countries? Eur J Obstet Gynecol Reprod Biol. 2011;154(2):177-181. doi:10.1016/j. ejogrb.2010.10.007

31. Jonard S, Robert Y, Dewailly D. Revisiting the ovarian volume as a diagnostic criterion for polycystic ovaries. Hum Reprod. 2005;20 (10):2893-2898. doi:10.1093/humrep/dei159

32. Dewailly D, Gronier H, Poncelet E, et al. Diagnosis of polycystic ovary syndrome (PCOS): revisiting the threshold values of follicle count on ultrasound and of the serum AMH level for the definition of polycystic ovaries. Hum Reprod. 2011;26(11):3123-3129. doi:10.1093/humrep/der297

33. Rao P, Bhide P. Controversies in the diagnosis of polycystic ovary syndrome. Ther Adv Reprod Health. 2020;14:2633494120913032.

34. Saxena U, Ramani M, Singh P. Role of AMH as diagnostic tool for polycystic ovarian syndrome. J Obstet Gynaecol India. 2018;68 (2):117-122. doi:10.1007/s13224-017-1066-4

35. Sahmay S, Aydin Y, Oncul M, Senturk LM. Diagnosis of polycystic ovary syndrome: AMH in combination with clinical symptoms. J Assist Reprod Genet. 2014;31(2):213-220. doi:10.1007/s10815013-0149-0

36. Zadehmodarres S, Heidar Z, Razzaghi Z, Ebrahimi L, Soltanzadeh K, Abed F. Anti-müllerian hormon level and polycystic ovarian syndrome diagnosis. Iran J Reprod Med. 2015;13(4):227-230. 
37. Wiweko B, Maidarti M, Priangga MD, et al. Anti-müllerian hormone as a diagnostic and prognostic tool for PCOS patients. J Assist Reprod Genet. 2014;31(10):1311-1316. doi:10.1007/s10815-0140300-6

38. Iliodromiti S, Kelsey TW, Anderson RA, Nelson SM. Can antiMüllerian hormone predict the diagnosis of polycystic ovary syndrome? A systematic review and meta-analysis of extracted data. $J$ Clin Endocrinol Metab. 2013;98(8):3332-3340. doi:10.1210/ jc.2013-1393

39. Bleil ME, Gregorich SE, Adler NE, Sternfeld B, Rosen MP, Cedars MI. Race/ethnic disparities in reproductive age: an examination of ovarian reserve estimates across four race/ethnic groups of healthy, regularly cycling women. Fertil Steril. 2014;101 (1):199-207. doi:10.1016/j.fertnstert.2013.09.015

40. Iglesias C, Banker M, Mahajan N, Herrero L, Meseguer M, GarciaVelasco JA. Ethnicity as a determinant of ovarian reserve: differences in ovarian aging between Spanish and Indian women. Fertil Steril. 2014;102(1):244-249. doi:10.1016/j.fertnstert.2014.03.050

41. Le MT, Le VNS, Le DD, Nguyen VQH, Chen C, Cao NT. Exploration of the role of anti-mullerian hormone and LH/FSH ratio in diagnosis of polycystic ovary syndrome. Clin Endocrinol. 2019;90(4):579-585. doi:10.1111/cen.13934

42. Yoo JH, Kim HO, Cha SW, et al. Age specific serum anti-Müllerian hormone levels in 1298 Korean women with regular menstruation. Clin Exp Reprod Med. 2011;38(2):93-97. doi:10.5653/ cerm.2011.38.2.93

43. Erdem A, Erdem M, Biberoglu K, Hayit O, Arslan M, Gursoy R. Age-related changes in ovarian volume, antral follicle counts and basal FSH in women with normal reproductive health. $J$ Reprod Med. 2002;47(10):835-839.
44. Wallace WH, Kelsey TW. Ovarian reserve and reproductive age may be determined from measurement of ovarian volume by transvaginal sonography. Hum Reprod. 2004;19(7):1612-1617. doi:10.1093/hum$\mathrm{rep} / \mathrm{deh} 285$

45. Tal R, Seifer DB. Ovarian reserve testing: a user's guide. Am J Obstet Gynecol. 2017;217(2):129-140. doi:10.1016/j.ajog.2017.02.027

46. Sachdeva G, Gainder S, Suri V, Sachdeva N, Chopra S. Comparison of the different PCOS phenotypes based on clinical metabolic, and hormonal profile, and their response to clomiphene. Indian $J$ Endocrinol Metab. 2019;23(3):326-331. doi:10.4103/ijem. IJEM_30_19

47. Głuszak O, Stopińska-Głuszak U, Glinicki P, et al. Phenotype and metabolic disorders in polycystic ovary syndrome. ISRN Endocrinol. 2012;2012:569862. doi:10.5402/2012/569862

48. Zhang S-D, Zhang H-L, Meng L. Compared the clinical and biochemical features of different phenotypic subtype of polycystic ovary syndrome. J Reprod Med. 2014;23:100-104.

49. Li H, Li L, Gu J, Li Y, Chen X, Yang D. Should all women with polycystic ovary syndrome be screened for metabolic parameters?: a hospital-based observational study. PLoS One. 2016;11(11): e0167036. doi:10.1371/journal.pone.0167036

50. Clark NM, Podolski AJ, Brooks ED, et al. Prevalence of polycystic ovary syndrome phenotypes using updated criteria for polycystic ovarian morphology: an assessment of over 100 consecutive women self-reporting features of polycystic ovary syndrome. Reprod Sci. 2014;21(8):1034-1043. doi:10.1177/1933719114522525
International Journal of Women's Health

\section{Publish your work in this journal}

The International Journal of Women's Health is an international, peerreviewed open-access journal publishing original research, reports, editorials, reviews and commentaries on all aspects of women's healthcare including gynecology, obstetrics, and breast cancer. The
Dovepress

manuscript management system is completely online and includes a very quick and fair peer-review system, which is all easy to use. Visit http://www.dovepress.com/testimonials.php to read real quotes from published authors. 